\title{
Results of a randomized, double blind, placebo controlled, crossover trial of melatonin for treatment of Nocturia in adults with multiple sclerosis (MeNiMS)
}

\author{
Marcus J. Drake ${ }^{1,2^{*}}$ D, Luke Canham ${ }^{3}$, Nikki Cotterill ${ }^{2}$, Debbie Delgado ${ }^{2}$, Jenny Homewood ${ }^{3}$, Kirsty Inglis ${ }^{3}$,
} Lyndsey Johnson ${ }^{2}$, Mary C. Kisanga², Denise Owen ${ }^{3}$, Paul White ${ }^{4}$ and David Cottrell ${ }^{3}$

\begin{abstract}
Background: Nocturia is a common urinary symptom of multiple sclerosis (MS) which can affect quality of life (QoL) adversely. Melatonin is a hormone known to regulate circadian rhythm and reduce smooth muscle activity such as in the bladder. There is limited evidence supporting use of melatonin to alleviate urinary frequency at night in the treatment of nocturia. The aim of this study was to evaluate the effect of melatonin on the mean number of nocturia episodes per night in patients with MS.

Methods: A randomized, double blind, placebo controlled crossover trial was conducted. 34 patients with nocturia secondary to multiple sclerosis underwent a 4-day pre-treatment monitoring phase. The patients were randomized to receive either $2 \mathrm{mg}$ per night (taken at bedtime) of capsulated sustained-release melatonin (Circadin ${ }^{\oplus}$ ) or 1 placebo capsule for 6 weeks followed by a crossover to the other regimen for an additional 6 weeks after a 1-month washout period.

Results: From the 26 patients who completed the study, there was no significant difference observed in the signs or symptoms of nocturia when taking $2 \mathrm{mg}$ melatonin compared to placebo. The primary outcome measure, mean number of nocturia episodes on bladder diaries, was 1.8/night at baseline, and 1.4/night on melatonin, compared with 1.6 for placebo (Medians 1.70, 1.50, and 1.30 respectively, $p=0.85$ ). There was also no significant difference seen in LUTS, QoL and sleep quality when taking melatonin. No significant safety concerns arose.
\end{abstract}

Conclusions: This small study suggests that a low dose of melatonin taken at bedtime may be ineffective therapy for nocturia in MS.

Trial registration: (EudraCT reference) 2012-00418321 registered: 25/01/13. ISRCTN Registry: ISRCTN38687869.

Keywords: Nocturia, Multiple sclerosis, Melatonin, Antidiuretic, Antimuscarinic, LUTS

\section{Background}

Nocturia is the complaint that the individual has to wake at night one or more times to void [1]. It can result from a range of factors, including behavioural influences, sleep disturbances, lower urinary tract dysfunction and altered fluid or salt homeostasis. Nocturia is prevalent in the general population and is known to increase in severity

\footnotetext{
* Correspondence: marcus.drake@bui.ac.uk

${ }^{1}$ School of Clinical Sciences, University of Bristol, Bristol, UK

${ }^{2}$ Bristol Urological Institute, Southmead Hospital, Bristol BS10 5NB, UK

Full list of author information is available at the end of the article
}

with age. $77 \%$ of people aged 60 and above experience some degree of nocturia with no difference seen between men and women [2]. Nocturia impacts greatly on quality of life (QoL), potentially due to fatigue, cognitive dysfunction and disturbed emotional health [3]. Furthermore, severe nocturia may be associated with cardiovascular disease, autonomic disease, obstructive sleep apnoea and chronic kidney disease [4], and potentially a higher risk of mortality [5]. A very high proportion of MS patients have lower urinary tract symptoms (LUTS) [6]. LUTS are a substantial problem in MS, and nocturia is a particularly

(c) The Author(s). 2018 Open Access This article is distributed under the terms of the Creative Commons Attribution 4.0 International License (http://creativecommons.org/licenses/by/4.0/), which permits unrestricted use, distribution, and 
prominent symptom with substantial detrimental impact on QoL [7].

Current treatments for nocturia include managing fluid intake, timed diuretics, desmopressin, antimuscarinic drugs, bedtime sedatives and miscellaneous compounds [8]. Desmopressin is indicated for treatment of nocturia in MS [9]. However, desmopressin can cause hyponatraemia [10] and has been associated with hyponatraemic convulsions $[11,12]$. Indeed, it is recommended that tri-cyclic antidepressants (commonly used in MS patients) are avoided when using Desmopressin to reduce the risk of hyponatraemia (British National Formulary). This can also potentially be an issue with diuretics. Antimuscarinics are known to cause dry mouth, constipation, swallowing difficulty and confusion. Patients taking sedatives can experience hangover sedation, while elderly subjects are at risk of cognitive impairment [13]. These side effects and poor efficacy mean that clinicians are sometimes reluctant to initiate treatment for nocturia.

Melatonin (N-acetyl-5-methoxytryptamine) is a hormone secreted primarily at night by the pineal gland. It regulates circadian rhythms and reduces smooth muscle spontaneous activity, including that found in the bladder [14]. Melatonin tablets taken before bedtime may reduce nocturia in a subgroup of patients with benign prostate enlargement [15]. In elderly patients with nocturia, levels of severity and QoL improve with melatonin use [16]. In MS, sleep quality is commonly reduced as a consequence of a wide range of sleep abnormalities, of which nocturia is only one example. In MS there can be an impairment of endogenous melatonin secretion $[17,18]$, and administration of oral melatonin improves reduced sleep quality in MS patients [19].

We hypothesised that bedtime administration of a melatonin sustained-release tablet will improve clinical nocturia in patients with MS. We previously published the protocol of the "Melatonin for nocturia in MS (MeNiMS)" study to evaluate this hypothesis [20], and the current study reports the findings. We chose a low dose of $2 \mathrm{mg}$, as melatonin levels negatively correlate with multiple sclerosis activity in humans, and alterations in endogenous melatonin have been proposed potentially to affect MS relapses [21]. The primary aim was to evaluate the effect of melatonin on mean number of nocturia episodes per night in MS patients. The secondary aims included: 1) improvement in QoL, 2) safety, 3) LUTS, 4) sleep quality and 5) total voided (urinated) volume and mean volume per void. A qualitative study was also included, which will be reported separately.

\section{Methods}

The detailed study protocol has previously been published [20]. In brief, male and female patients aged $\geq 18$ were recruited at Southmead Hospital, Bristol, UK. Each patient had a confirmed neurological diagnosis of MS as per the 2010 McDonald MS Criteria [22]. They also reported at least one episode of nocturia every night on the International Consultation on Incontinence Questionnaires (ICIQ) Nocturia questionnaire (ICIQ-N [23]). Patients were excluded if they had (i) an indwelling urinary catheter; (ii) used desmopressin or investigational medications in the month preceding randomization; (iii) taken antimuscarinic or diuretic medication, unless used long-term prior to study ( $>3$ months) and continued throughout the study; (iv) taken melatonin on prescription or purchased; (v) used "sleeping tablets" on prescription or purchased; (vi) diabetes mellitus/diabetes insipidus; and (vii) or if they were pregnant at screening, or of child-bearing potential and unwilling to use contraception. Dipstick urinalysis to exclude urinary tract infection was undertaken at screening. The U.K. National Research Ethics Service Committee South West - Exeter approved the study protocol (REC reference number: 12/ SW/0322).

This was a randomized, double blind, placebo controlled crossover trial with two groups (Fig. 1). Following an initial four-day pre-treatment monitoring phase, the treatment phases were $2 \mathrm{mg}$ per night of capsulated sustained-release melatonin ('Circadin') or an identical placebo capsule per night for 6 weeks each, separated by a 4 week washout period. Patients were allocated double-blind via a website (http://www.randomization.com) to group $\mathrm{AB}$ or $\mathrm{BA}$, with unblinding undertaken following database lock and analysis ( $\mathrm{A}=$ placebo, $\mathrm{B}=$ melatonin).

The primary outcome was reduction in nocturia episodes per night, derived from the ICIQ bladder diary (ICIQ-BD) [24]. Secondary outcomes included; 1 . Subjective severity, using the ICIQ tools on nocturia (ICIQ-N) and nocturia quality of life (ICIQ-NQoL) [25]. 2. MS quality of life, assessed with the MSQoL scale. 3. LUTS, assessed with the ICIQ-MLUTS and ICIQ-FLUTS for males and females respectively. 4. Sleep quality; measured with the Pittsburgh Sleep Quality Index (PSQI). 5. Safety, based on adverse event reporting and Expanded Disability Status Scale (EDSS) score [26]. Outcome measures were completed at baseline and at the end of each treatment phase. Adverse event reporting was undertaken throughout, and followed up until resolution or for 3 months [20].

\section{Statistical analysis}

We calculated that for a two-sided test, using standard levels of statistical significance (alpha $=0.05$ ), a sample size of $n=21$ complete data sets would have $80 \%$ power to detect a medium to large effect size (Cohen's $d=0.65$ ) with $80 \%$ power, and a sample size of $n=34$ would be needed for a medium effect size (Cohen's $d=0.5)$ [20].

The balanced two group, two period, two sequence, double-blind, randomised crossover design with wash-out 


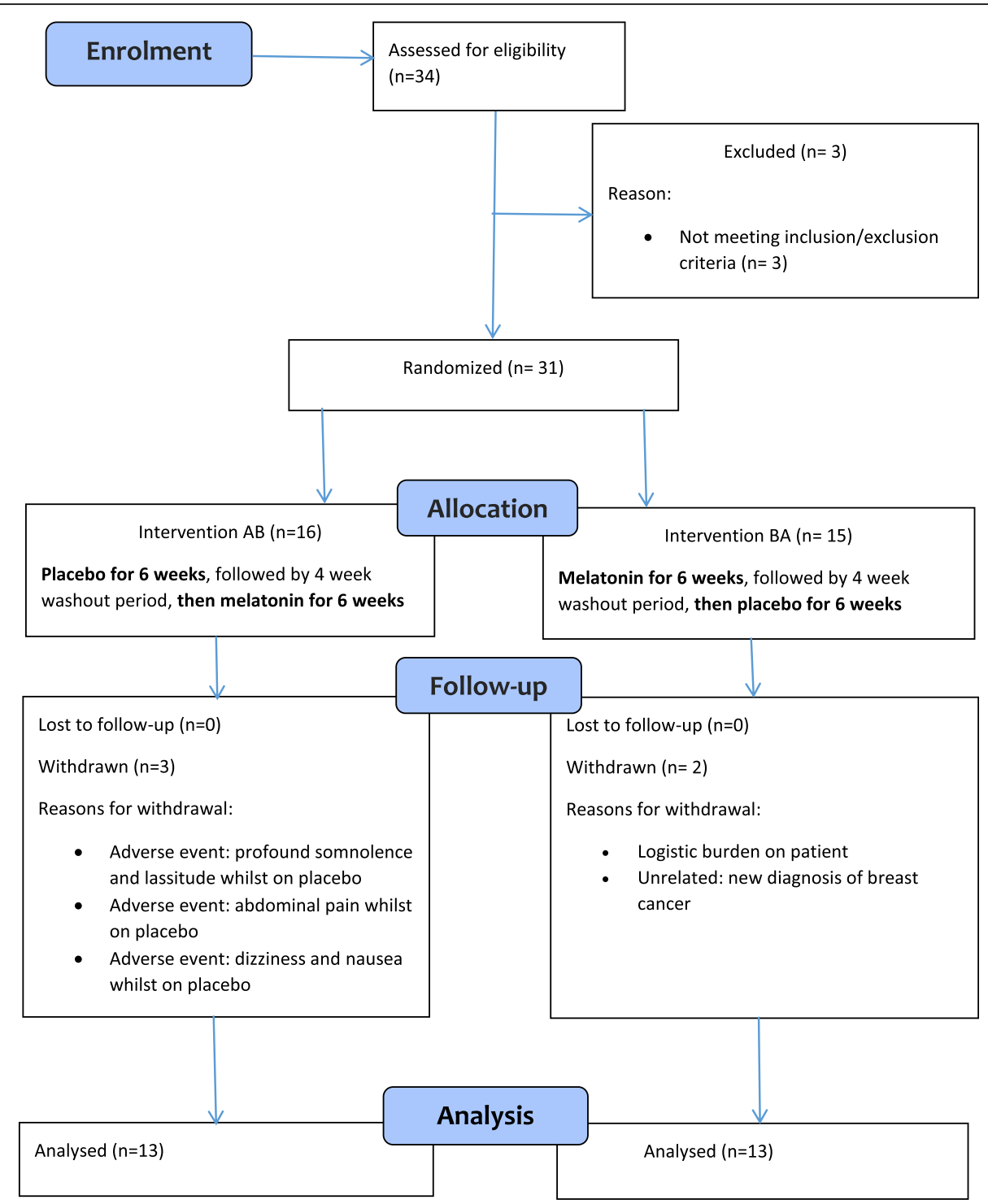

Fig. 1 Study flow chart

period comparing treatment to control, ranks highly in the hierarchy of designs. The analyses of the resultant data under this $\mathrm{AB} / \mathrm{BA}$ design may proceed using nonparametric (Mann-Whitney-Wilcoxon $U$ ) two-sample statistical techniques which assess for carryover effects, period effects, and treatment effect accounting for any period effects using independent samples designs [27] or using paired samples in the absence of period and carryover effects. Treatment effect sizes have been quantified and converted to Cohen's $d$. For Cohen's standardized statistic, $d=0$ indicates the absence of an effect. For statistically significant effects, some broad and cautious threshold guidance to aid interpretation is for, $0<d<0.1$ to indicate a trivial effect, $0.1<d<0.3$ to indicate a small effect, $0.3<d<0.5$ to indicate a moderate effect, $0.5<d<$ 0.8 a medium size effect, $0.8<d<1.3$ a large effect, and $d>1.3$ a very large effect [28]. A missing data analysis was also performed on the primary outcome measure to assess sensitivity of statistical conclusions to missingness. This analysis indicated any missing data to be consistent with being missing completely at random (MCAR). Multiple Imputation by Chained Equations (MICE) [29] with 1000 imputed data sets was performed. These imputed analyses faithfully reproduced the findings from the observed sample data and for brevity of exposition, and to avoid redundancy, are not reported in full.

\section{Results}

In total 13 men and 18 women of mean age 54.8 years (range 34-69) were randomised. Five patients had Primary Progressive MS, 15 patients had Secondary Progressive MS and 11 patients had Relapsing Remitting MS (RRMS). 
Five of the 11 with RRMS were taking disease modifying therapy at the time of the trial (one interferon beta-1a, two fingolomid and two dimethyl fumarate). Mean EDSS was 4.2 (median 4.0 , range 1.5 to 8.0 ). Five patients withdrew from the trial (Fig. 1). Reasons for withdrawal were; adverse events (three patients), new-onset unrelated health problems (one patient) and logistic burden (one patient). Mean nocturia severity at baseline from the bladder diary was 1.78 episodes/night and self-reported mean ICIQ symptom score for nocturia was 1.80 episodes/night (range 1-3).

The effects of $2 \mathrm{mg}$ melatonin and placebo on bladder diary parameters are shown in Table 1 . There was no significant change seen with melatonin for the primary outcome measure, the number of nocturnal episodes per night. "Objective" nocturia was 1.4/ night for melatonin, compared with 1.6 for placebo $(\mathrm{U}=43, p=0.85)$. Average nocturnal output and nocturnal polyuria index (NPI) were also not significantly altered. Effects on patient-reported LUTS are shown in Table 2. For patient-reported (subjective) nocturia, the number of episodes per night after 6 weeks of melatonin was 3.3 , compared with 3.2 with placebo. Overall scores and individual LUTS were not significantly different with melatonin compared with placebo.

Secondary end points looking at QoL also revealed that melatonin did not significantly affect outcomes. The ICIQ-NQoL questionnaire mean totals were 22.1 for melatonin, compared with 23.6 for placebo $(U=41.5$, $p=0.34$; Table 3). For the MSQoL scale (Table 4), there was no significant change between melatonin and placebo in most of the domains, except for physical overall score (50.9 vs. 47.9, respectively, median 48.5 vs 44.0 , $U=26, p=0.02$ ), role limitations due to physical problems (39.1 vs. 33.3 , median 25 vs $12.5, U=29, \mathrm{p}=0.02$ ) and pain score ( 62.7 vs. 70.6 , median 70 vs. $78, U=34.5$, $p=0.03$ ). In the PSQI (Table 5), the mean scores were 8.1 and 8.7 for melatonin and placebo respectively (median 8.0 vs. 9.0 respectively, $U=56.5, p=0.89$ ).

In all analyses there was little evidence of any carryover effects (i.e. no evidence of period by treatment interaction effects, consistent with a sufficiently large washout period). In addition, the given conclusions for treatment effects are replicated if differences are examined as paired differences using the non-parametric Wilcoxon signed rank test.

\section{Safety}

EDSS data is given in Table 6. Overall, there was no difference in EDSS while taking melatonin or placebo; mean score was 4.4 for placebo and 4.7 for melatonin (medians 4.0 and 4.0). Four patients reported worsening symptoms of MS during the study, of which two were taking melatonin at the time. One experienced two separate episodes, once whilst taking melatonin and once whilst taking placebo. One patient experienced Uhthoff's phenomenon, a worsening of neurological condition related to MS, while taking melatonin.

Adverse event reporting most commonly identified urinary tract infection (UTI), which affected seven participants. Four of these were prior to receipt of study medication. Three UTIs were found after randomisation. One was on treatment phase 1 and was receiving melatonin. Another had completed treatment phase 2 (placebo). One participant was found to have a UTI after the post drug wash out phase for melatonin.

Two patients experienced faecal urgency; both were no longer taking study medication at the time of onset, and both had been taking placebo.

Two participants experienced lassitude and anergia. One was withdrawn by the clinician, and was found to have been taking placebo. The other had already completed the study, and had been taking melatonin as the most recent study medication.

One patient experienced severe dizziness, and was unblinded and was found to have been taking placebo; this patient was withdrawn from the study. A further patient reported abdominal pain (a reported potential side effect of Melatonin), and was withdrawn by the clinician without unblinding; subsequently the patient was found to have been taking placebo.

Two patients reported chest infections, one taking placebo and the other melatonin. One of these patients went on to report a further three adverse events of pain in fingers, knees and shoulder, which all occurred whilst taking placebo.

Table 1 Median and range for average number of nocturia episodes, voided output, and NPI by treatment with treatment $p$-value and standardized effect size, $d$

\begin{tabular}{|c|c|c|c|c|c|c|c|c|}
\hline \multirow[t]{3}{*}{ Measure } & \multicolumn{8}{|c|}{ Treatment } \\
\hline & \multicolumn{2}{|c|}{ Baseline } & \multicolumn{2}{|c|}{ A: Placebo } & \multicolumn{2}{|c|}{ B: Melatonin } & \multirow[t]{2}{*}{$p$} & \multirow[t]{2}{*}{$d$} \\
\hline & Median & Range & Median & Range & Median & Range & & \\
\hline Average Nocturia Episodes & 1.70 & $1-3$ & 1.50 & $0-3$ & 1.30 & $0-3.3$ & 0.618 & 0.136 \\
\hline Average nocturnal urine output (mls) & 741 & $310-1416$ & 651 & $300-1933$ & 667 & $200-1100$ & 0.939 & 0.020 \\
\hline 24-h voided volume (mls) & 2125 & $1200-4000$ & 2213 & $1250-3900$ & 2000 & $881-3600$ & 0.254 & 0.314 \\
\hline Nocturnal Polyuria Index NPI & 0.32 & $0.15-0.71$ & 0.33 & $0.17-0.56$ & 0.32 & $0.15-0.60$ & 0.849 & 0.052 \\
\hline
\end{tabular}


Table 2 Median and range of the grouped sub-scores (Voiding and Incontinence) and the individual symptom scores for nocturia, urgency and frequency from the ICIQ LUTS questionnaires (MLUTS and FLUTS for males and females respectively) by treatment, with $p$-values and standardized effect size, $d$

\begin{tabular}{|c|c|c|c|c|c|c|}
\hline \multirow[t]{3}{*}{ Measure } & \multicolumn{6}{|c|}{ Treatment } \\
\hline & \multicolumn{2}{|c|}{ A: Placebo } & \multicolumn{2}{|c|}{ B: Melatonin } & \multirow[t]{2}{*}{$p$} & \multirow[t]{2}{*}{$d$} \\
\hline & Median & Range & Median & Range & & \\
\hline Voiding sub-score & 4.0 & $0-12$ & 2.0 & $0-12$ & 0.096 & 0.492 \\
\hline Incontinence (Storage) sub-score & 3.0 & $0-9$ & 3.0 & $0-12$ & 0.107 & 0.450 \\
\hline Nocturia & 2.0 & $1-3$ & 2.0 & $1-3$ & 0.892 & 0.037 \\
\hline Urgency & 2.0 & $0-3$ & 2.0 & $1-4$ & 0.772 & 0.079 \\
\hline
\end{tabular}

One patient experienced abdominal pain resulting in emergency department review, where cholecystitis was diagnosed. She also reported shingles and reduced mobility in separate adverse events. She had been taking the placebo on all of these occasions. One patient reported a probable olecranon bursitis while taking placebo. Colds and an ear infection were reported by two patients; both were taking placebo.

\section{Discussion}

Nocturia is a prominent symptom with substantial detrimental impact on quality of life in MS, especially in light of the range of factors affecting sleep quality in these patients. Ordinarily, there should be a reduction in urine production rate during sleep. Endogenous melatonin is a key contributor in circadian control, and disruption of melatonin is a feature in sleep disturbance in MS [30]. We surmised that beneficial effects may result indirectly by improved sleep quality, and perhaps directly by some restoration of the normal circadian reduction in urine production at night, and reduced bladder smooth muscle activity. A potential impact of giving supplementary melatonin orally as regulator of circadian rhythms in restoring some measure of circadian control [31] could be beneficial in the proposed context. In reality, the effect of melatonin in the current study did not identify any reduction in nocturia (either objectively or subjectively). The bladder diary was the main outcome assessment, and during the active treatment phase there was no reduction in nocturia episodes or overall nocturnal urine production.

For the PSQI, the mean scores at the end of the treatment phase were 8.1 for melatonin and 8.7 for placebo.
This difference was statistically significant, and seems to indicate worse function with melatonin, but the difference is modest and unlikely to be clinically significant. We also evaluated other aspects of the patient's health and QoL. For the MSQoL, there was no significant change between melatonin and placebo in most of the domains, except that small statistically significant differences were evident in the physical overall score, role limitations due to physical problems, and pain score. It is unclear that this was a definite consequence of melatonin action.

Symptom scores were a key secondary measure, and again melatonin did not appear to have any effect on nocturia specifically or LUTS in general. Nocturia-specific quality of life did not show any evident improvement. Nocturia has a multifactorial pathophysiology, which potentially could mean that melatonin might have effect in some individuals and not others, and this was considered to explain the finding of a responder group in a previous study which examined melatonin use for treatment of nocturia in men with benign prostate enlargement [15]. However, there did not seem to be any evident responder group in the current study.

The lack of evident difference between melatonin and placebo may reflect the pragmatic approach taken in the study. Diabetes mellitus and diabetes insipidus were excluded, but otherwise inclusive criteria were used for study recruitment. This pragmatic approach was taken to reflect the utility of a therapy which could be applied to the majority of patients in the general healthcare context, without having to undertake too much clinical assessment. The fact that we did not identify benefit could reflect the wide range of potential co-morbidity in

Table 3 Median and range for ICIQ NQol by treatment with p-value and standardized effect size, $d$

\begin{tabular}{|c|c|c|c|c|c|c|}
\hline \multirow[t]{3}{*}{ Measure } & \multicolumn{6}{|c|}{ Treatment } \\
\hline & \multicolumn{2}{|c|}{ A: Placebo } & \multicolumn{2}{|c|}{ B: Melatonin } & \multirow[t]{2}{*}{$p$} & \multirow[t]{2}{*}{$d$} \\
\hline & Median & Range & Median & Range & & \\
\hline Overall score & 25.0 & $8-35$ & 20.0 & $8-42$ & 0.341 & 0.261 \\
\hline Overall interference & 5.0 & $0-9$ & 4.0 & $0-9$ & 0.444 & 0.209 \\
\hline Sleep/Energy sub-score & 11.0 & $3-19$ & 10.0 & $3-18$ & 0.549 & 0.164 \\
\hline Bother/Concern sub-score & 9.5 & $4-21$ & 9.0 & $3-16$ & 0.288 & 0.292 \\
\hline
\end{tabular}


Table 4 Median and range for MSQoL by treatment, with $p$-value and standardized effect size, $d$

\begin{tabular}{|c|c|c|c|c|c|c|}
\hline \multirow[t]{3}{*}{ Measure } & \multicolumn{6}{|c|}{ Treatment } \\
\hline & \multicolumn{2}{|c|}{ A: Placebo } & \multicolumn{2}{|c|}{ B: Melatonin } & \multirow[t]{2}{*}{$p$} & \multirow[t]{2}{*}{$d$} \\
\hline & Median & Range & Median & Range & & \\
\hline Mental Overall Score & 68.0 & $16-92$ & 75.0 & $28-92$ & 0.110 & 0.446 \\
\hline Physical Overall Score & 44.0 & $17-89$ & 48.5 & $21-90$ & 0.023 & 0.651 \\
\hline Energy Score & 30.0 & $0-76$ & 36.0 & $0-84$ & 0.212 & 0.347 \\
\hline Emotional Wellbeing & 68.0 & $32-100$ & 72.0 & $32-100$ & 0.961 & 0.013 \\
\hline Physical Health & 45.0 & $5-100$ & 40.0 & $5-100$ & 0.701 & 0.105 \\
\hline Role limitations due to physical problems & 12.5 & $0-100$ & 25.0 & $0-100$ & 0.015 & 0.701 \\
\hline Role limitations due to emotional problems & 100 & $0-100$ & 100 & $0-100$ & 0.580 & 0.151 \\
\hline Health Perceptions & 42.5 & $5-85$ & 45.0 & $0-80$ & 0.396 & 0.233 \\
\hline Social Function & 67.0 & $25-100$ & 67.0 & $0-100$ & 0.603 & 0.142 \\
\hline Cognitive Function & 67.0 & $0-100$ & 73.0 & $0-100$ & 0.884 & 0.040 \\
\hline Health Distress & 60.0 & $15-90$ & 60.0 & $0-100$ & 0.862 & 0.036 \\
\hline Change in Health & 25.0 & $0-50$ & 50.0 & $25-100$ & 0.647 & 0.125 \\
\hline Quality of Life & 55.0 & $27-95$ & 63.0 & $0-90$ & 0.130 & 0.421 \\
\hline Pain Score & 78.0 & 0-100 & 70.0 & $0-100$ & 0.029 & 0.622 \\
\hline
\end{tabular}

MS, indicating that it is likely that assessment of specific underlying mechanisms probably is needed to understand nocturia in MS. A low dose of melatonin was chosen $(2 \mathrm{mg})$, because at the time the study was designed there was discussion that melatonin could potentially contribute to deterioration in MS severity through effects on immune function [32]. Based on the results, it seems that this low dose was insufficient for nocturia therapy, even though it is a standard dose for treating sleep disorders in a wider population. Some studies have used higher doses of melatonin in an MS population, demonstrating improved sleep quality [19]. Recent literature does not identify detrimental effect of melatonin on MS severity, and alternative hypotheses have been promulgated regarding a potential beneficial effect. We did not measure endogenous melatonin production, or effective serum melatonin levels on treated patients, so we are unable to state whether serum levels of the hormone reached therapeutic levels in our study population.

The inclusion of a placebo group is an essential part of LUTS investigation, since placebo responses are noted to be rather big generally [33]. In the current study, the placebo response seen in the bladder diaries and symptom scores was modest. Undertaking observations such as bladder diaries is considered a potential factor that could influence patient behaviour, since completing a bladder diary shows to a patient when they are generating a high urine output, which can feed back on their behaviour. This was not a particular observation in the current study, where the baseline $24 \mathrm{~h}$ voided volume was $2.2 \mathrm{~L}$, and was similar during the treatment phases (2.2 $\mathrm{L}$ for placebo, and $2.0 \mathrm{~L}$ per $24 \mathrm{~h}$ for melatonin, medians $2.1 \mathrm{~L}, 2.2 \mathrm{~L}$, and $2.0 \mathrm{~L}$ respectively). The nocturnal polyuria index was unchanged (median 0.32, $0.33,0.32$

Table 5 Median and range for PSQI by treatment, with $p$-value and standardized effect size, $d$

\begin{tabular}{|c|c|c|c|c|c|c|}
\hline \multirow[t]{3}{*}{ Measure } & \multicolumn{6}{|c|}{ Treatment } \\
\hline & \multicolumn{2}{|c|}{ A: Placebo } & \multicolumn{2}{|c|}{ B: Melatonin } & \multirow[t]{2}{*}{$p$} & \multirow[t]{2}{*}{$D$} \\
\hline & Median & Range & Median & Range & & \\
\hline Overall Score & 9.0 & $2-13$ & 8.0 & $2-16$ & 0.893 & 0.036 \\
\hline Sleep Disturbances & 2.0 & $1-3$ & 2.0 & $1-3$ & 0.092 & 0.471 \\
\hline Sleep Medication & 0.0 & $0-0$ & 0.0 & $0-3$ & 0.228 & 0.333 \\
\hline Sleep Duration & 1.0 & $0-3$ & 1.0 & $0-3$ & 0.663 & 0.119 \\
\hline Sleep Latency & 1.0 & $0-5$ & 1.0 & $0-3$ & 0.927 & 0.025 \\
\hline Sleep Quality & 1.0 & $0-3$ & 1.0 & $0-3$ & 0.922 & 0.027 \\
\hline Daytime Dysfunction & 1.0 & $0-3$ & 1.0 & $1-3$ & 0.141 & 0.410 \\
\hline Habitual Sleep Efficiency & 2.0 & $0-3$ & 1.0 & $0-3$ & 0.114 & 0.440 \\
\hline
\end{tabular}


Table 6 Median and range for EDSS by treatment with $p$-value and standardized effect size, $d$

\begin{tabular}{|c|c|c|c|c|c|c|}
\hline \multirow[t]{3}{*}{ Measure } & \multicolumn{6}{|c|}{ Treatment } \\
\hline & \multicolumn{2}{|c|}{ A: Placebo } & \multicolumn{2}{|c|}{ B: Melatonin } & \multirow[t]{2}{*}{$p$} & \multirow[t]{2}{*}{$d$} \\
\hline & Median & Range & Median & Range & & \\
\hline Visual & 1.0 & $0-4$ & 1.5 & $0-4$ & 0.056 & 0.539 \\
\hline Brainstem & 1.0 & $0-2$ & 1.0 & $0-2$ & 0.288 & 0.292 \\
\hline Sensory & 3.0 & $0-4$ & 3.0 & $0-3$ & 0.090 & 0.474 \\
\hline Pyramidal & 2.0 & $0-3$ & 2.0 & $0-3$ & 0.386 & 0.238 \\
\hline Cerebellar & 2.0 & $1-4$ & 2.0 & $0-3$ & 0.181 & 0.370 \\
\hline Cerebral & 2.0 & $0-3$ & 2.0 & $0-3$ & 0.653 & 0.123 \\
\hline Bladder and Bowel & 2.0 & $1-3$ & 2.0 & $0-3$ & 0.674 & 0.115 \\
\hline Ambulatory & 1.0 & $0-9$ & 1.0 & $0-9$ & 1.00 & 0.000 \\
\hline EDSS & 4.0 & $1.5-8$ & 4.0 & $3-8$ & 0.209 & 0.347 \\
\hline
\end{tabular}

for baseline, placebo and melatonin respectively). A small reduction in median nocturnal urine output was seen (baseline $741 \mathrm{~mL}$, placebo $651 \mathrm{~mL}$, and melatonin $667 \mathrm{~mL}$ ), but this was not statistically significant. It did not yield a significant change in nocturia episodes (median $1.70,1.50$ and 1.30 respectively).

There was no clear adverse safety signal. No adverse events appeared to have any clear link to the melatonin therapy on a consistent basis. UTIs were reported, and two episodes of faecal urgency. Single presentations with Uhthoff's phenomenon, feeling drained, cold hands, profound somnolence, and others were also described. The qualitative interviews also identified that fatigue was a key feature. The individual with Uhthoff's phenomenon explains the difference in the visual domain on the EDSS scores, which were otherwise not significantly different for the melatonin and placebo phases.

\section{Conclusions}

In summary, a low dose of melatonin taken at bedtime may be an ineffective therapy for nocturia in MS, studying an adult population with nocturia once per night or more often. A different dose regime of melatonin or recruitment selection criteria would need to considered to ascertain whether melatonin could influence nocturia in MS.

\footnotetext{
Abbreviations

CaMBS: Cambridge Multiple Sclerosis Basic Score; EDSS: Expanded Disability Status Scale; FVC: Frequency volume chart; ICIQ: International Consultation on Incontinence Questionnaires; ICIQ-FLUTS: Female- specific tool for assessing severity and bother of all LUTS; ICIQ-MLUTS: Male-specific tool for assessing severity and bother of all LUTS; ICIQ-N: International Consultation on Incontinence-Nocturia; ICIQ-NQoL: A nocturia-specific quality of life tool which will be used throughout the study; LUTS: Lower urinary tract symptoms; MeNiMS: Study Acronym: Melatonin for Nocturia in Multiple Sclerosis; MS: Multiple sclerosis; MSQLI: Multiple sclerosis quality of life index; PSQI: Pittsburgh Sleep Quality Index
}

\section{Acknowledgements}

Neurim and Flynn Pharma; unrestricted donation of study medication.

\section{Funding}

Funded by a Research Project Grant from the MS Society, UK (Grant 959/11) to cover all aspects of setting up, running and analysing the trial.

\section{Availability of data and materials}

Supporting data can be accessed by contacting the corresponding author.

\section{Authors' contributions}

MD Chief Investigator; LC recruitment, data collection; NC data collection, qualitative review; DD ethics application, drafted the manuscript; $\mathrm{JH}$ recruitment; KI recruitment, EDSS testing; $L$ recruitment, data collection; MCK data analysis, drafted the manuscript; DO recruitment, data collection; PW statistical analysis; DC recruitment, drafted the manuscript. All authors read and approved the final manuscript.

\section{Ethics approval and consent to participate}

The U.K. National Research Ethics Service Committee South West - Exeter approved the study protocol (REC reference number: 12/SW/0322).

\section{Consent for publication}

Not applicable.

\section{Competing interests}

MJD; Research grants, Speaker bureau, advisory boards for Allergan, Astellas, Ferring. Speaker bureau for Hikma and Pfizer. Other authors; not applicable.

\section{Publisher's Note}

Springer Nature remains neutral with regard to jurisdictional claims in published maps and institutional affiliations.

\section{Author details}

${ }^{1}$ School of Clinical Sciences, University of Bristol, Bristol, UK. ${ }^{2}$ Bristol Urological Institute, Southmead Hospital, Bristol BS10 5NB, UK. ${ }^{3}$ Neurology Department, Southmead Hospital, Bristol BS10 5NB, UK. ${ }^{4}$ University of the West of England, Bristol, UK.

Received: 10 August 2017 Accepted: 1 August 2018

Published online: 06 August 2018

\section{References}

1. Abrams P, Cardozo L, Fall M, Griffiths D, Rosier P, Ulmsten U, van Kerrebroeck $P$, Victor A, Wein A. The standardisation of terminology of lower urinary tract function: report from the standardisation sub-committee of the international continence society. Neurourol Urodyn. 2002;21(2):167-78.

2. Bing $M H$, Moller LA, Jennum $P$, Mortensen $S$, Skovgaard LT, Lose G. Prevalence and bother of nocturia, and causes of sleep interruption in a Danish population of men and women aged 60-80 years. BJU Int. 2006; 98(3):599-604

3. Stanley N. The underestimated impact of nocturia on quality of life. Eur Urol Suppl. 2005:4:17-9.

4. Gulur DM, Mevcha AM, Drake MJ. Nocturia as a manifestation of systemic disease. BJU Int. 2011:107(5):702-13.

5. Lightner DJ, Krambeck AE, Jacobson DJ, McGree ME, Jacobsen SJ, Lieber MM, Roger VL, Girman CJ, St Sauver JL. Nocturia is associated with an increased risk of coronary heart disease and death. BJU Int. 2012;110(6):848-53.

6. Chancellor MB, Blaivas JG. Urological and sexual problems in multiple sclerosis. Clin Neurosci. 1994;2(3-4):189-95.

7. Stanton BR, Barnes F, Silber E. Sleep and fatigue in multiple sclerosis. Mult Scler. 2006;12(4):481-6.

8. Marshall SD, Raskolnikov D, Blanker MH, Hashim H, Kupelian V, Tikkinen KA, Yoshimura K, Drake MJ, Weiss JP. International consultations on urological D: Nocturia: current levels of evidence and recommendations from the international consultation on male lower urinary tract symptoms. Urology. 2015;85(6):1291-9

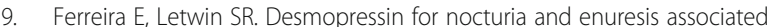
with multiple sclerosis. Ann Pharmacother. 1998;32(1):114-6.

10. Friedman FM, Weiss JP. Desmopressin in the treatment of nocturia: clinical evidence and experience. Ther Adv Urol. 2013;5(6):310-7. 
11. Hamed M, Mitchell H, Clow DJ. Hyponatraemic convulsion associated with desmopressin and imipramine treatment. BMJ. 1993;306(6886):1169.

12. Larney V, Dwyer R. Hyponatraemic convulsions and fatal head injury secondary to desmopressin treatment for enuresis. Eur J Anaesthesiol. 2006;23(10):895-7.

13. Nelson J, Chouinard G. Guidelines for the clinical use of benzodiazepines: pharmacokinetics, dependency, rebound and withdrawal. Canadian Society for Clinical Pharmacology. Can J Clin Pharmacol. 1999;6(2):69-83.

14. Gomez-Pinilla PJ, Gomez MF, Sward K, Hedlund P, Hellstrand P, Camello PJ Andersson KE, Pozo MJ. Melatonin restores impaired contractility in aged Guinea pig urinary bladder. J Pineal Res. 2008:44(4):416-25.

15. Drake MJ, Mills IW, Noble JG. Melatonin pharmacotherapy for nocturia in men with benign prostatic enlargement. J Urol. 2004;171(3):1199-202.

16. Sugaya K, Nishijima S, Miyazato M, Kadekawa K, Ogawa Y. Effects of melatonin and rilmazafone on nocturia in the elderly. J Int Med Res. 2007; 35(5):685-91.

17. Damasceno A, Moraes AS, Farias A, Damasceno BP, dos Santos LM, Cendes F. Disruption of melatonin circadian rhythm production is related to multiple sclerosis severity: a preliminary study. J Neurol Sci. 2015;353(1-2): $166-8$.

18. Melamud L, Golan D, Luboshitzky R, Lavi I, Miller A. Melatonin dysregulation, sleep disturbances and fatigue in multiple sclerosis. J Neurol Sci. 2012; 314(1-2):37-40.

19. Adamczyk-Sowa M, Pierzchala K, Sowa P, Mucha S, Sadowska-Bartosz I, Adamczyk J, Hartel M. Melatonin acts as antioxidant and improves sleep in MS patients. Neurochem Res. 2014:39(8):1585-93.

20. Delgado D, Canham L, Cotterill N, Cottrell D, Drake MJ, Inglis K, Owen D, White P. Protocol for a randomized, double blind, placebo controlled, crossover trial of melatonin for treatment of Nocturia in adults with multiple sclerosis (MeNiMS). BMC Neurol. 2017;17(1):63.

21. Farez MF, Mascanfroni ID, Mendez-Huergo SP, Yeste A, Murugaiyan G, Garo $L P$, Balbuena Aguirre ME, Patel B, Ysrraelit MC, Zhu C, et al. Melatonin contributes to the seasonality of multiple sclerosis relapses. Cell. 2015;162(6): 1338-52.

22. Polman CH, Reingold SC, Banwell B, Clanet M, Cohen JA, Filippi M, Fujihara K, Havrdova E, Hutchinson M, Kappos L, et al. Diagnostic criteria for multiple sclerosis: 2010 revisions to the McDonald criteria. Ann Neurol. 2011;69(2): 292-302.

23. Abrams P, Avery K, Gardener N, Donovan J. The international consultation on incontinence modular questionnaire: www.iciq.net. J Urol. 2006;175(3 Pt 1):1063-6. discussion 1066

24. Bright E, Cotterill N, Drake M, Abrams P. Developing and validating the international consultation on incontinence questionnaire bladder diary. Eur Urol. 2014:66(2):294-300

25. Abraham L, Hareendran A, Mills IW, Martin ML, Abrams P, Drake MJ, MacDonagh RP, Noble JG. Development and validation of a quality-of-life measure for men with nocturia. Urology. 2004;63(3):481-6.

26. Kurtzke JF. The disability status scale for multiple sclerosis: apologia pro DSS sua. Neurology. 1989;39(2 Pt 1):291-302.

27. Wellek $\mathrm{S}$, Blettner M. On the proper use of the crossover design in clinical trials: part 18 of a series on evaluation of scientific publications. Dtsch Arztebl Int. 2012;109(15):276-81.

28. Ellis PD: The essential guide to effect sizes statistical power, meta-analysis, and the interpretation of research results. Cambridge University press; 2010.

29. Azur MJ, Stuart EA, Frangakis C, Leaf PJ. Multiple imputation by chained equations: what is it and how does it work? Int J Methods Psychiatr Res. 2011;20(1):40-9.

30. Barun B. Pathophysiological background and clinical characteristics of sleep disorders in multiple sclerosis. Clin Neurol Neurosurg. 2013;115(Suppl 1):S82-5.

31. Cardinali DP, Cano P, Jimenez-Ortega V, Esquifino Al. Melatonin and the metabolic syndrome: physiopathologic and therapeutical implications. Neuroendocrinology. 2011;93(3):133-42.

32. Sandyk R. Multiple sclerosis: the role of puberty and the pineal gland in its pathogenesis. Int J Neurosci. 1993;68(3-4):209-25.

33. Eredics $K$, Madersbacher $\mathrm{S}$, Schauer I. A relevant mid-term (12 months) placebo effect on lower urinary tract symptoms and maximum flow rate in male LUTS/BPH - a meta-analysis. Urology. 2017.

\section{Ready to submit your research? Choose BMC and benefit from:}

- fast, convenient online submission

- thorough peer review by experienced researchers in your field

- rapid publication on acceptance

- support for research data, including large and complex data types

- gold Open Access which fosters wider collaboration and increased citations

- maximum visibility for your research: over $100 \mathrm{M}$ website views per year

At BMC, research is always in progress.

Learn more biomedcentral.com/submissions 